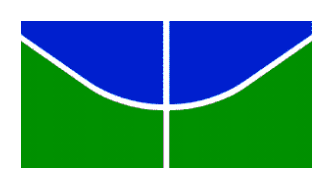

UNIVERSIDADE DE BRASÍLIA

Faculdade de Agronomia e Medicina Veterinária

Programa de Pós-Graduação em Saúde Animal

\title{
AVALIAÇÃO DE PARÂMETROS PERFUSIONAIS NAS \\ DIFERENTES CLASSES DE INSUFICIÊNCIA CARDÍACA EM \\ CÃES
}

FELIPE BORGES SOARES

DISSERTAÇÃO DE MESTRADO

EM SAÚDE ANIMAL

BRASÍLIA/DF

FEVEREIRO/2015 


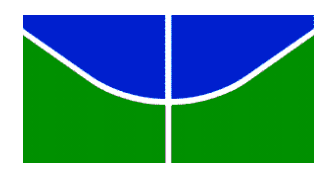

UNIVERSIDADE DE BRASÍLIA

Faculdade de Agronomia e Medicina Veterinária

Programa de Pós-Graduação em Saúde Animal

\section{AVALIAÇÃO DE PARÂMETROS PERFUSIONAIS NAS DIFERENTES CLASSES DE INSUFICIÊNCIA CARDÍACA EM CÃES}

FELIPE BORGES SOARES

ORIENTADORA: GLÁUCIA BUENO PEREIRA NETO

PUBLICAÇÃO: 106/2015

DISSERTAÇÃO DE MESTRADO EM SAÚDE ANIMAL ÁREA DE CONCENTRAÇÃO: CLÍNICA MÉDICA E CIRURGIA ANIMAL LINHA DE PESQUISA: MÉTODOS DE DIAGNÓSTICO E TRATAMENTO DE AFECÇÕES DOS ANIMAIS DOMÉSTICOS E SILVESTRES

BRASÍLIA/DF

FEVEREIRO/2015 


\section{AVALIAÇÃo DE PARÂMETROS PERFUSIONAIS NAS DIFERENTES CLASSES DE INSUFICIÊNCIA CARDÍACA EM CÃES}

FELIPE BORGES SOARES

DISSERTAÇÃO DE MESTRADO
SUBMETIDA AO PROGRAMA DE PÓS-
GRADUAÇÃO EM SAÚDE ANIMAL,
COMO PARTE DOS REQUISITOS
NECESSÁRIOS À OBTENÇÃO DO GRAU
DE MESTRE EM SAÚDE ANIMAL.

APROVADA POR:

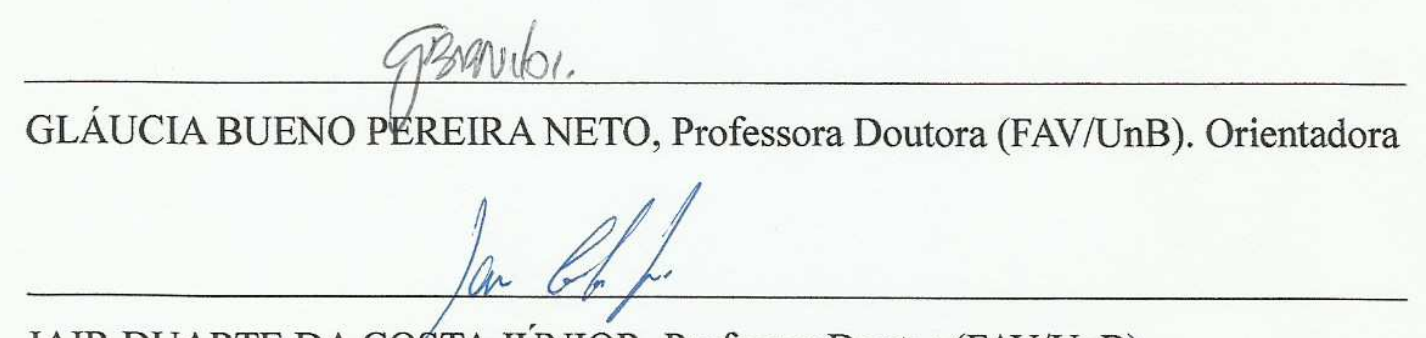

JAIR DUARTE DA COSTA JÚNIOR, Professor Doutor (FAV/UnB)

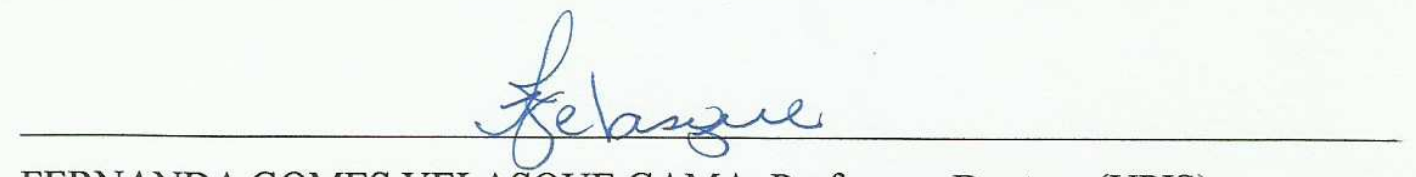

FERNANDA GOMES VELASQUE GAMA, Professora Doutora (UPIS)

BRASÍLIA/DF, 09 DE FEVEREIRO DE 2015 


\title{
REFERÊNCIA BIBLIOGRÁFICA E CATALOGAÇÃO
}

SOARES, F. B. Avaliação de parâmetros perfusionais nas diferentes classes de insuficiência cardíaca em cães. Brasília: Faculdade de Agronomia e Medicina Veterinária, Universidade de Brasília, 2015, 36 p. Dissertação de Mestrado.

\begin{abstract}
Documento formal, autorizando reprodução desta dissertação de mestrado para empréstimo ou comercialização, exclusivamente para fins acadêmicos, foi passado pelo autor à Universidade de Brasília e acha-se arquivado na Secretaria do Programa. O autor reserva para si os outros direitos autorais, de publicação. Nenhuma parte desta dissertação de mestrado pode ser reproduzida sem a autorização por escrito do autor. Citações são estimuladas, desde que citada a fonte.
\end{abstract}

Soares, Felipe Borges

Avaliação de parâmetros perfusionais nas diferentes classes de insuficiência cardíaca em cães/Felipe Borges Soares

Orientação de Gláucia Bueno Pereira Neto

Brasília, 2015. 36 p. : il.

Dissertação de Mestrado (M) - Universidade de Brasília/Faculdade de Agronomia e Medicina Veterinária, 2015.

1. Canino. 2. Insuficiência Cardíaca. 3. Perfusão.

4. Hiperlactatemia. I. SOARES, F.B. II. Título 


\section{AGRADECIMENTOS}

A Deus, por sempre ter me apoiado na minha caminhada.

À minha esposa, que mesmo longe sempre esteve presente em todos os momentos da minha vida desde que nos conhecemos.

À minha família, por ter me educado valorizando a grandeza de caráter e a educação mais do que tudo.

À minha orientadora, Profa Dra. Gláucia Bueno Pereira Neto, por ter acredito em meu potencial, mesmo antes de me conhecer.

Ao Dr. Rodrigo Cardoso Rabelo, por toda a orientação e apoio na condução deste trabalho.

À equipe do Hospital Veterinário de Pequenos Animais da Universidade de Brasília, pela ajuda na condução deste experimento.

À equipe do Batalhão de Polícia do Exército de Brasília, por possibilitar a realização desta importante etapa da minha vida. 


\section{SUMÁRIO}

\section{Página}

Lista de abreviaturas..................................................................................... vii

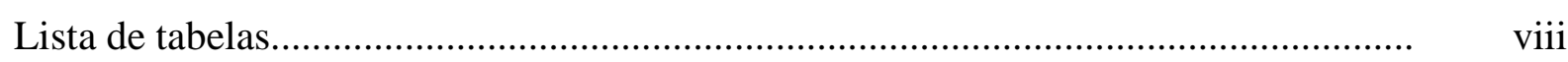

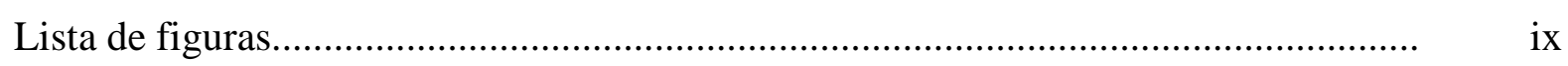

Informações adicionais.................................................................................... $\mathrm{x}$

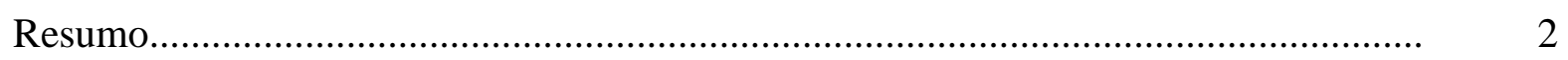

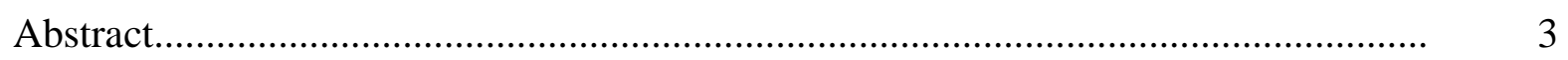

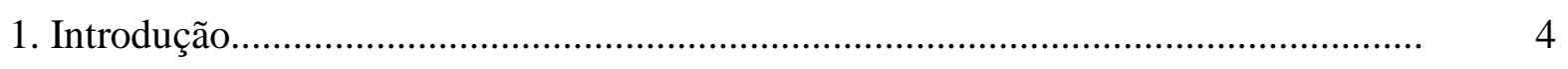

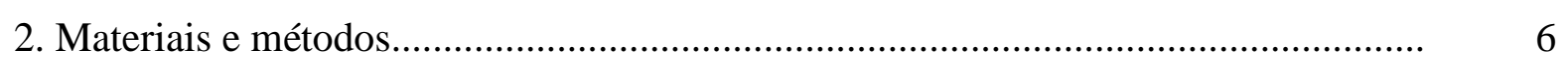

2.1 Animais e grupos experimentais................................................................. 6

2.2 Diagnóstico da doença valvar e estadiamento da insuficiência cardíaca............. 7

2.3 Avaliação dos parâmetros clínicos e laboratoriais............................................... 8

2.4 Análise estatística..................................................................................... 8

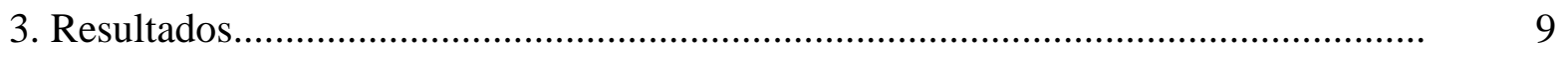

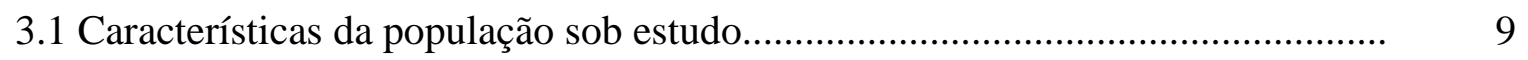

3.2 Parâmetros de exame físico, perfusionais e hematológicos.................................. 11

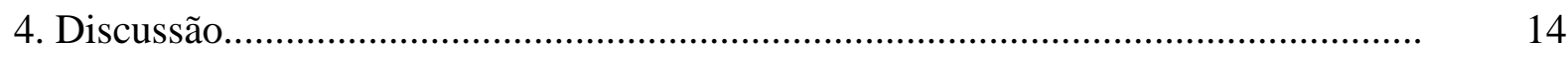

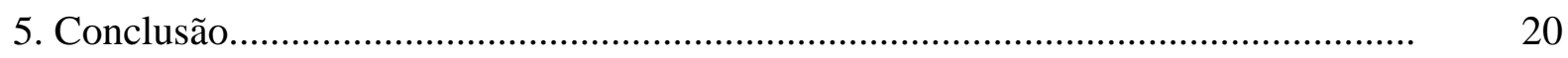

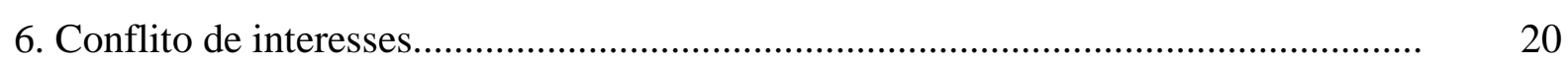

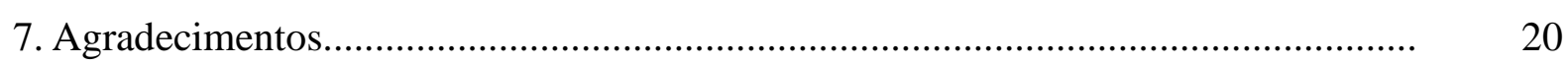

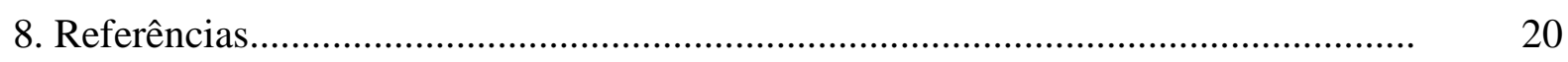

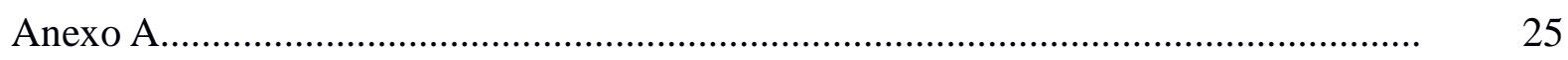

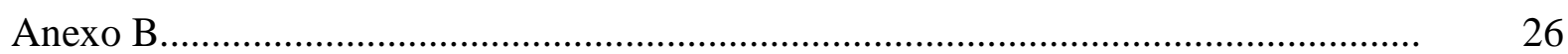




\section{LISTA DE ABREVIATURAS}

ACC

$\mathrm{AE}$

AHA

Ao

ATP

bpm

CEUA

$\mathrm{CO}_{2}$

DCVM

DP

$\mathrm{g} / \mathrm{dL}$

IC

ICC

IECA

IIQ

ISACHC

$\mathrm{kg}$

$\mathrm{mm}^{3}$

$\mathrm{mmHg}$

$\mathrm{mmol} / \mathrm{L}$

mrm

PAS

PPT

SRAA

$\mathrm{SvcO}_{2}$

$\mathrm{SvO}_{2}$

$\mu \mathrm{L}$

${ }^{\circ} \mathrm{C}$

$\Delta \mathrm{Tcp}$

$\Delta \mathrm{Tpa}$
American College of Cardiology

Átrio esquerdo

American Heart Association

Aorta

Adenosina trifosfato

Batimentos por minuto

Comissão de Ética no Uso Animal

Dióxido de carbono

Doença Crônica da Valva Mitral

Desvio-padrão

Gramas por decilitro

Insuficiência cardíaca

Insuficiência cardíaca congestiva

Inibidor da enzima conversora de angiotensina

Intervalo interquartil

International Small Animal Cardiac Health Council

Quilograma

Milímetros cúbicos

Milímetros de mercúrio

Milimol por litro

Movimentos respiratórios por minuto

Pressão arterial sistêmica sistólica

Proteína plasmática total

Sistema renina, angiotensina, aldosterona

Saturação venosa central de oxigênio

Saturação venosa mista de oxigênio

Microlitro

Graus Celsius

Gradiente entre as temperaturas central e periférica

Gradiente entre as temperaturas periférica e ambiente 


\section{LISTA DE TABELAS}

\section{Página}

Tabela 1. Aspectos relativos a sexo, idade, peso e raça dos cães pertencentes aos diferentes grupos experimentais.

Tabela 2. Distribuição das medicações administradas aos animais dos diferentes grupos experimentais

Tabela 3. Descrição do grau dos sopros apresentados pelos animais nos diferentes grupos experimentais.

Tabela 4. Parâmetros de exame físico, perfusionais e hematológicos nos diferentes grupos experimentais 


\section{LISTA DE FIGURAS}

Página

Figura 1. Organograma de seleção de cães com DCVM para inclusão no estudo, com

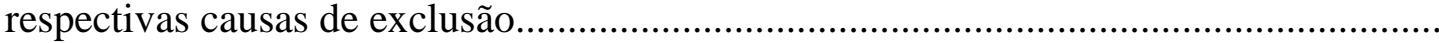

Figura 2. Gráfico box-plot representando os níveis de lactato (mmol/L) nos diferentes grupos experimentais. 


\section{INFORMAÇÕES ADICIONAIS}

A presente dissertação encontra-se formatada segundo as normas de submissão de artigos para publicação no periódico The Veterinary Journal, classificado como B1 no sistema Qualis/CAPES. 
Avaliação de parâmetros perfusionais nas diferentes classes de insuficiência cardíaca em cães

F.B. Soares ${ }^{\mathrm{a}, *}$, G.B. Pereira-Neto ${ }^{\mathrm{a}}$, R.C. Rabelo ${ }^{\mathrm{b}}$

${ }^{a}$ Hospital Veterinário da Faculdade de Agronomia e Medicina Veterinária, Universidade de Brasília, Av. L4 Norte, Setor de Clubes Norte, Campus Universitário Darcy Ribeiro, Brasília, DF 70910-900, Brasil.

${ }^{\mathrm{b}}$ Intensivet Veterinary Consulting, Brasília, DF 70864-110, Brasil.

*Autor para correspondência. Tel.: 556182545352

Endereço de e-mail: fbsborges@ hotmail.com (F.B. Soares). 


\section{Resumo}

O objetivo do estudo foi avaliar os parâmetros perfusionais de cães com insuficiência cardíaca decorrente da doença crônica da valva mitral, visando determinar se há alterações que sigam um padrão de acordo com a evolução do estágio da doença e desenvolvimento da insuficiência cardíaca, segundo o sistema de estadiamento modificado da American Heart Association e do American College of Cardiology (AHA/ACC). Adotou-se a hipótese de que cães com insuficiência cardíaca podem apresentar hipoperfusão tecidual oculta detectável por meio de parâmetros clínicos e laboratoriais. Foram recrutados 146 animais e após ajuste para os critérios de exclusão, foram selecionados cães classificados no estágio B2 (média \pm desviopadrão, idade 11,29 \pm 1,79 anos; peso $11,84 \pm 4,21 \mathrm{~kg}, \mathrm{n}=7$ ) e no estágio $\mathrm{C}$ (média \pm desviopadrão, idade 13,50 \pm 1,84 anos; peso $6,00 \pm 3,02 \mathrm{~kg}, \mathrm{n}=10$ ) de insuficiência cardíaca de acordo com AHA/ACC. Seis cães que não apresentavam DCVM foram avaliados e compuseram o grupo Controle (média \pm desvio-padrão, idade $9 \pm 2$ anos; peso 7,62 $\pm 2,32$ kg). Dentre os parâmetros avaliados, o lactato sérico apresentou valores mais elevados no Estágio C (mediana, intervalo interquartil; 3,70, 3,30-4,02 mmol/L) quando comparados aos grupos Controle (mediana, intervalo interquartil; 2,80, 2,55-3,35 $\mathrm{mmol} / \mathrm{L})(P=0,024)$ e Estágio B2 (mediana, intervalo interquartil; 2,70, 2,00-3,80 mmol/L) $(P=0,045)$. O gradiente de temperatura centro-periférico também apresentou uma tendência importante do ponto de vista clínico, sendo maior no grupo Estágio B2 que no Estágio C, apesar de não gerar diferença estatisticamente significativa. Conclui-se que cães em insuficiência cardíaca decorrente de DCVM podem apresentar hipoperfusão tecidual oculta no Estágio C, constatada pela hiperlactatemia. A insuficiência cardíaca deve ser considerada no diagnóstico diferencial de hiperlactatemia em pacientes com DCVM.

Palavras-chave: Canino; Choque oculto; Hiperlactatemia; Insuficiência Cardíaca; Lactato; Perfusão. 


\section{Abstract}

The objective of the study was to evaluate the perfusion parameters in dogs with heart failure caused by myxomatous mitral valve disease (MMVD), to determine whether there are changes that follow a pattern according to the evolution of the stage of the disease and development of heart failure, according to the American Heart Association and the American College of Cardiology (AHA/ACC) modified classification system. The hypothesis adopted was that dogs with heart failure may have hidden tissue hypoperfusion detectable by clinical and laboratory parameters. We recruited 146 animals and, after adjusting for the exclusion criteria, dogs with heart failure classified in stage B2 (mean \pm standard deviation, age $11.29 \pm$ 1.79 years; weight $11.84 \pm 4.21 \mathrm{~kg}, \mathrm{n}=7$ ) and stage $\mathrm{C}$ (mean \pm standard deviation, age $13.50 \pm$ 1.84 years; weight $6.00 \pm 3.02 \mathrm{~kg}, \mathrm{n}=10$ ) according to AHA/ACC were selected. Six dogs with no MMVD were evaluated and composed the Control group (mean \pm standard deviation, age $9 \pm 2$ years; weight $7.62 \pm 2.32 \mathrm{~kg}$ ). Among the evaluated parameters, serum lactate showed higher values in Stage C (median, interquartile range, 3.70, 3.30 to $4.02 \mathrm{mmol} / \mathrm{L}$ ) when compared to Control (median, interquartile range, $2.80,2.55$ to $3.35 \mathrm{mmol} / \mathrm{L})(P=$ $0.024)$ and Stage B2 groups (median, interquartile range, $2.70,2.00$ to $3.80 \mathrm{mmol} / \mathrm{L})(P=$ 0.045). The core-peripheral temperature gradient also showed an important trend from the clinical point of view, being higher in Stage B2 group than in Stage C, although statistically significant difference was not noted. It was concluded that dogs in heart failure due to MMVD may have hidden tissue hypoperfusion in Stage $\mathrm{C}$, evidenced by hyperlactatemia. Heart failure should be considered in the differential diagnosis of hyperlactatemia in patients with MMVD.

Keywords: Canine; Cryptic Shock; Hyperlactatemia; Heart Failure; Lactate; Perfusion. 


\section{Introdução}

A Doença Crônica da Valva Mitral (DCVM) é a cardiopatia adquirida mais frequente em cães e está caracterizada pela destruição do colágeno associada à deposição de mucopolissacárides nas camadas espongiosa e fibrosa da valva (Borgarelli e Buchanan, 2012). Este processo promove o espessamento e a formação de irregularidades na superfície valvar, o que dificulta a coaptação congruente e ocasiona insuficiência com a regurgitação da mitral (Borgarelli e Haggstrom, 2010; Olsen et al., 2010). Sugere-se que cães de pequeno porte com regurgitação mitral grave decorrente da DCVM apresentem insuficiência cardíaca (IC) motivada principalmente pelo intenso fluxo regurgitante (Kittleson e Brown, 2003).

Cães com insuficiência cardíaca congestiva (ICC) evidenciada apresentam elevações nos níveis séricos de aldosterona e na atividade plasmática da renina, o que corrobora a ativação do sistema renina, angiotensina, aldosterona (SRAA). O aumento de volume plasmático devido a retenção de sódio e água e a diminuição da capacitância venosa por venoconstrição, ambos decorrentes desta ativação, induzem ao aumento da pressão venosa e consequente congestão pulmonar ou sistêmica, que prejudica a perfusão tecidual em grau superior (Sisson, 2010).

A avaliação da perfusão tecidual pode ser realizada de maneira subjetiva por meio dos parâmetros clínicos hemodinâmicos centrais como a pressão arterial sistêmica, a frequência cardíaca e a qualidade de pulso arterial palpado. Porém, é sabido que em muitas situações, caso haja diminuição do débito cardíaco, a pressão arterial sistêmica pode ser mantida por vasoconstrição periférica (Wadell, 2010). Assim sendo, os tecidos periféricos estarão submetidos a uma situação de hipóxia no indivíduo que apresenta pressão arterial sistêmica, nível de consciência e temperatura central inalterados, situação denominada hipoperfusão oculta (Hu et al., 2012).

Acredita-se que o prejuízo à perfusão tecidual possa ser elucidado mais precocemente, 
mesmo antes do aparecimento de manifestações clínicas da IC, por meio da mensuração de indicadores de perfusão tecidual utilizados também de forma fácil na prática clínica, como os gradientes de temperatura e o lactato sérico (Boag e Hughes, 2005; Lima e Bakker, 2005).

A utilização de gradientes de temperatura como parâmetros de perfusão periférica, principalmente em pacientes sob terapia intensiva, teve início com o estudo que avaliou a temperatura periférica em humanos como indicador de choque circulatório (Joly e Weil, 1969). A vasoconstrição promove diminuição de temperatura periférica, o que faz com que o monitoramento da temperatura nos órgãos menos vitais possa ser um marcador precoce de hipoperfusão (Lima e Bakker, 2005; van Genderen et al., 2012).

Diversas vias metabólicas atuam na formação e no "clearance" do lactato, e uma das principais é a disfunção adquirida da cadeia respiratória mitocondrial que pode ser causada por hipóxia tecidual. Neste estado, as células tornam-se dependentes da glicólise citosólica, e esta via de produção de ATP não requer oxigênio, mas haverá produção de lactato por meio da redução do piruvato, e que comumente resulta em hiperlactatemia (Adeva-Andany et al., 2014). A hipoperfusão tecidual oculta associada à hiperlactatemia foi estudada em pacientes humanos com IC, porém os autores desconhecem artigos publicados que verifiquem sua ocorrência em cães com ICC (Ander et al., 1999a; Ander et al., 1999b).

O objetivo primário desse estudo prospectivo observacional foi avaliar os parâmetros clínicos perfusionais em cães com IC decorrente da doença crônica da valva mitral, visando determinar se há alterações que sigam um padrão de acordo com a evolução do estágio da doença e desenvolvimento da IC, segundo o sistema de estadiamento da IC da American Heart Association e do American College of Cardiology (AHA/ACC) modificado (Atkins et al., 2009). Adotou-se a hipótese de que cães com IC podem apresentar hipoperfusão tecidual oculta detectável precocemente por meio de parâmetros de avaliação da perfusão periférica e que indiquem uma correta relação entre o metabolismo aeróbio e anaeróbio nos diferentes 
estágios de IC.

\section{Materiais e métodos}

\subsection{Animais e grupos experimentais}

A população sob estudo foi composta por cães provenientes da rotina de atendimento do Hospital Veterinário Universitário de Pequenos Animais da Universidade de Brasília, avaliados de agosto a novembro de 2014. Realizou-se uma seleção prospectiva de cães saudáveis e cães com DCVM sob tratamento, entre machos e fêmeas, adultos, idosos e de raças de pequeno porte e foram estabelecidos os seguintes critérios de exclusão do estudo: (1) a presença de doenças concomitantes como doença renal crônica, hepatopatias, diabetes mellitus, lesões de pele, doenças respiratórias, doenças ortopédicas, trauma nos últimos 30 dias, sepse, anemia, neoplasias e epilepsia (2) fêmeas gestantes ou no cio, de acordo com o relato do proprietário; (3) uso de terapia com corticosteróides no momento da avaliação; (4) ausência de termo de consentimento assinado pelo proprietário. Todos os critérios de exclusão utilizados foram baseados no objetivo de evitar qualquer outra causa de ativação simpática compensatória ou hiperlactatemia que não fossem geradas pela doença cardíaca em curso (Boysen et al., 2009; Lima et al., 2010; Rabelo, 2010; Strehlow, 2010; van Hall, 2010; AdevaAndany et al., 2014). Os cães foram divididos em três grupos: Controle, Estágio B2 e Estágio C de acordo com a ausência ou presença de DCVM e com o estadiamento clínico da IC baseado no sistema modificado da AHA/ACC (Anexo A) (Atkins et al., 2009). Um único operador realizou as avaliações clínicas e exames complementares nos animais do estudo.

O estudo foi aprovado pela Comissão de Ética no Uso Animal (CEUA) do Instituto de Ciências Biológicas da Universidade de Brasília, UnBDOC nº 45831/2013, em 7 de junho de 2013. Todos os proprietários expressaram por escrito sua concordância em ceder seus animais 
para participar do projeto.

\subsection{Diagnóstico da doença valvar e estadiamento da insuficiência cardíaca}

A avaliação cardiológica de todos os cães de cada grupo experimental foi composta por anamnese, exame físico e o exame ecodopplercardiográfico somente com a finalidade de diagnosticar a doença valvar e classificar os pacientes nos diferentes estágios de IC. Os cães do grupo Controle eram assintomáticos de acordo com os dados da anamnese, não apresentaram sopro em nenhum dos focos cardíacos ao exame físico e observou-se a ausência de espessamento da valva mitral e de fluxo regurgitante no átrio esquerdo por meio de visualização com doppler colorido e doppler contínuo ao exame ecodopplercardiográfico. Os animais dos grupos Estágio B2 (assintomáticos) e Estágio C (sintomáticos) foram submetidos ao exame ecodopplercardiográfico após a auscultação de sopro sistólico em foco mitral. Durante este exame observou-se a presença de espessamento dos folhetos da valva mitral e de fluxo regurgitante em átrio esquerdo por meio do doppler colorido e doppler contínuo. Avaliaram-se os diâmetros do átrio esquerdo e da aorta no modo bidimensional, com a posterior determinação da relação AE/Ao, o diâmetro interno de ventrículo esquerdo em sístole e diástole no modo $\mathrm{M}$ e as frações de ejeção e encurtamento obtidas pelo método de Teichholz. Os animais incluídos nesses grupos apresentaram relação AE/Ao maior que 1,5 e, em alguns casos, aumento no diâmetro interno do ventrículo esquerdo em diástole e/ou sístole (Kienle, 1998). Para a obtenção das imagens nas diferentes modalidades ecocardiográficas utilizou-se um ecocardiógrafo (DC3-Vet, Mindray, Shenzhen, China) com um transdutor setorial. A diferenciação entre os grupos Estágio B2 e Estágio C foi baseada nos dados da anamnese e nas manifestações clínicas de dificuldade respiratória e/ou episódios documentados de expressão clínica de IC nos pacientes do estágio C. 


\subsection{Avaliação dos parâmetros clínicos e laboratoriais}

Todos os cães foram avaliados em um único momento, após o diagnóstico da DCVM ou a verificação de sua ausência. Efetuou-se a mensuração da pressão arterial sistólica não invasiva utilizando-se um sistema com doppler vascular (DV 610, Medmega, Franca, Brasil) e esfigmomanômetro (Gamma G5, Heine, Herrsching, Alemanha), de acordo com as diretrizes para a espécie (Brown et al., 2007). Com o cão em decúbito lateral direito, realizou-se a aferição no membro torácico esquerdo. O valor adotado, após descarte da primeira medida, foi a média aritmética de cinco medições consecutivas realizadas. Sequencialmente, foi realizado o registro das temperaturas ambiente e periféricas (temperatura interdigital), a fim de calcular o gradiente entre a temperatura central (retal) do paciente e periférica $(\Delta \mathrm{Tcp}$ baseada na temperatura do membro pélvico esquerdo) e o gradiente entre a temperatura periférica e do ambiente ( $\Delta$ Tpa). Por fim, amostras de sangue foram obtidas por punção da veia jugular externa para realização de hemograma, determinação das concentrações séricas de uréia, creatinina, bilirrubinas total, direta e indireta, albumina, proteína total, alanina aminotransferase, fosfatase alcalina e eletrólitos (potássio, cálcio e fósforo). Durante a punção venosa, o garrote foi liberado para a coleta do sangue a fim de minimizar possíveis interferências na mensuração dos níveis de lactato. No momento imediatamente após a coleta foram mensurados a glicemia e o lactato (Accutrend Plus, Roche, Basileia, Suíça). Animais cujos resultados de exames complementares indicaram a presença de comorbidade foram excluídos do experimento conforme previsto.

\subsection{Análise estatística}

Os dados obtidos foram avaliados utilizando-se o software GaphPad Prism 6, versão 6.05. A distribuição dos dados relativos às variáveis contínuas foi analisada através do teste Shapiro-Wilk e verificou-se que não apresentam distribuição Normal, sendo então expressos 
através de suas medianas e intervalos interquartis. O teste de Kruskal-Wallis para dados não paramétricos foi utilizado para a realização da comparação entre os grupos. O teste de Mann Withney bicaudal foi utilizado como teste post-hoc para a determinação pontual de diferenças intergrupos estatisticamente significativas verificadas previamente. Testes que apresentaram valores de $P<0,05$ foram considerados estatisticamente significativos.

\section{Resultados}

\subsection{Características da população sob estudo}

Dentre os 146 cães com DCVM acompanhados no período do estudo, 129 foram excluídos do estudo pelas razões explicitadas na Figura 1.

Figura 1. Organograma de seleção de cães com DCVM para inclusão no estudo, com respectivas causas de exclusão.

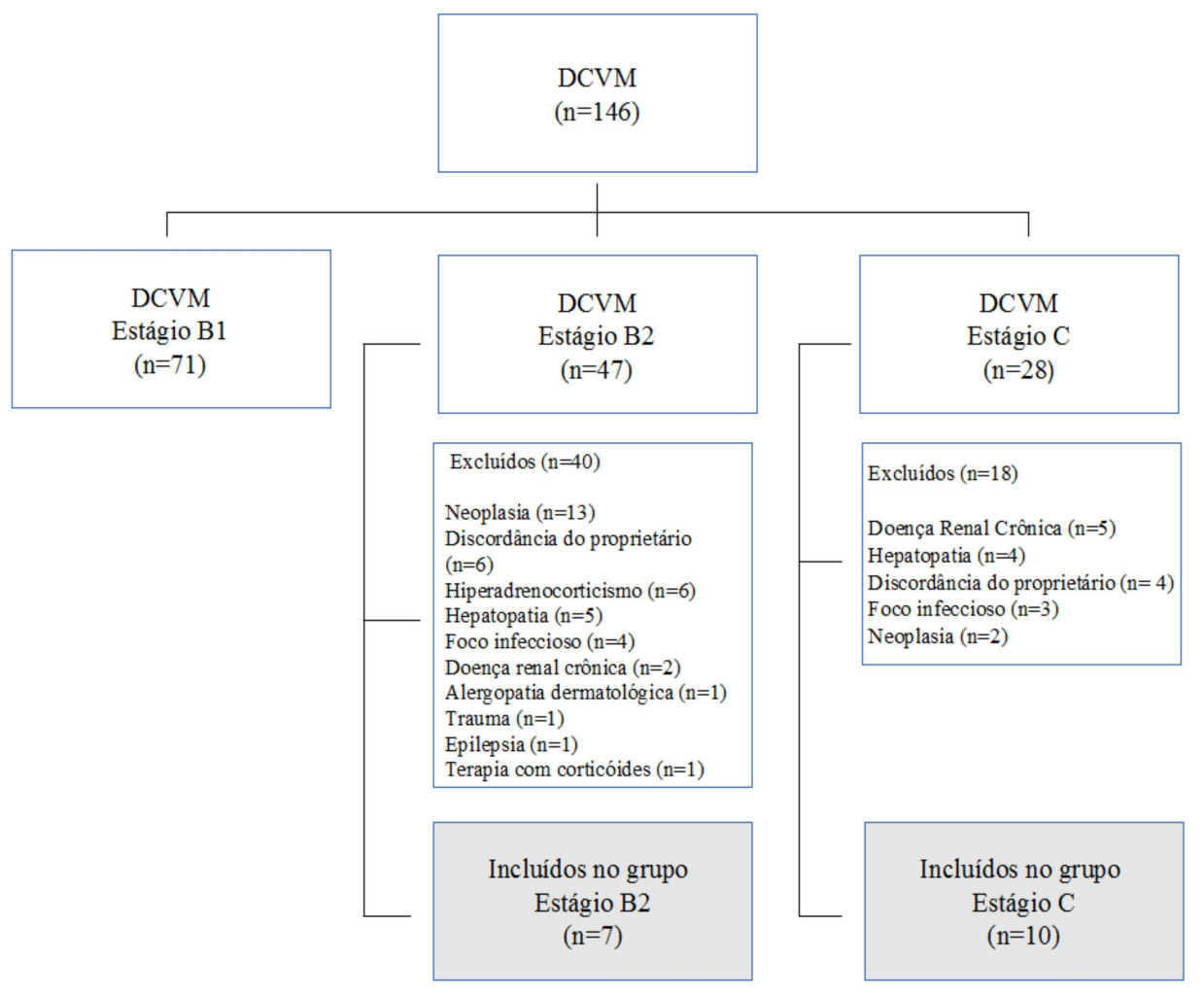

Legenda: DCVM: Doença crônica da valva mitral. 
A porcentagem de machos superou a de fêmeas em todos os grupos. Observou-se que os animais do estudo eram adultos e idosos, com idade superior a seis anos e peso corporal inferior a $20 \mathrm{~kg}$ (Goldston e Hoskins, 1999). Houve diferença estatisticamente significativa na avaliação da idade entre os grupos Controle e Estágio C $(P=0,001)$ e entre Estágio B2 e Estágio $\mathrm{C}(P=0,039)$. Em ambos os casos o grupo Estágio $\mathrm{C}$ apresentou idade superior aos demais grupos. Não houve diferença estatisticamente significativa na comparação do peso corporal entre os grupos. As informações acerca destas características, bem como o padrão de distribuição racial, podem ser encontrados na Tabela 1.

Tabela 1. Aspectos relativos ao sexo, idade, peso e raça dos cães pertencentes aos diferentes grupos experimentais.

\begin{tabular}{lccc}
\hline \multicolumn{1}{c}{ Características } & Controle $(\mathrm{n}=6)$ & Estágio B2 $(\mathrm{n}=7)$ & Estágio C $(\mathrm{n}=10)$ \\
\hline Sexo & & & \\
\hline Macho $(\%)$ & $67(4)$ & $57(4)$ & $80(8)$ \\
\hline Fêmea $(\%)$ & $33(2)$ & $43(3)$ & $20(2)$ \\
\hline Idade (anos; média \pm DP; amplitude) & $9 \pm 2 ; 6-11$ & $11,29 \pm 1,79 ; 9-14$ & $13,50 \pm 1,84 ; 10-16$ \\
\hline Peso (kg; média \pm DP; amplitude) & $7,62 \pm 2,32 ; 3,25-10,2$ & $11,84 \pm 4,21 ; 6,8-18,4$ & $6,00 \pm 3,02 ; 1,9-10$ \\
\hline Raça & & & 3 \\
\hline Poodle & 2 & 2 & 3 \\
\hline SRD & 2 & - & - \\
\hline Dachshund & 1 & - & 1 \\
\hline Yorkshire & 1 & 2 & - \\
\hline Cocker spaniel & - & 1 & - \\
\hline Terrier brasileiro & - & - & 3 \\
\hline Pinscher & - & 2 & 3 \\
\hline
\end{tabular}

Legenda: DP: desvio-padrão.

Os animais incluídos no projeto pertencentes ao grupo Controle não estavam sob tratamento no momento da avaliação. A distribuição das medicações recebidas pelos animais dos grupos Estágio B2 e Estágio C encontra-se representada na Tabela 2. A mediana dos tempos de tratamento foi de seis meses (IIQ = 1-9) e de sete meses (IIQ = 4,5-16,25) para os grupos Estágio B2 e Estágio C, respectivamente, sem diferença estatisticamente significativa 
entre eles $(P=0,721)$.

Tabela 2. Distribuição das medicações administradas aos animais dos diferentes grupos experimentais.

\begin{tabular}{lccccccc}
\hline \multirow{2}{*}{ Grupos (n) } & \multicolumn{7}{c}{ Tratamento n (\%) } \\
\cline { 2 - 8 } & IECA & Espironolactona & Furosemida & Pimobendan & Sildenafil & Codeína & Digoxina \\
\hline Controle (6) & 0 & 0 & 0 & 0 & 0 & 0 & 0 \\
Estágio B2 (7) & $7(100)$ & $7(100)$ & $1(14)$ & 0 & 0 & 0 & 0 \\
Estágio C (10) & $10(100)$ & $8(80)$ & $10(100)$ & $6(60)$ & $2(20)$ & $2(20)$ & $1(10)$ \\
\hline
\end{tabular}

Legenda: IECA: inibidor da enzima conversora da angiotensina.

\subsection{Parâmetros de exame físico, perfusionais e hematológicos}

Os cães pertencentes ao grupo Controle e Estágio B2 eram assintomáticos para IC. Já os animais do grupo Estágio C manifestavam tosse (100\%), intolerância a exercícios (40\%), engasgos (40\%), síncope (30\%), cianose (30\%), edema de membros $(10 \%)$ e cansaço fácil $(10 \%)$.

Ao exame físico, todos os animais apresentaram-se sem alterações em linfonodos palpáveis, sem evidência clínica de desidratação, com murmúrios vesiculares à auscultação pulmonar, com ausência de pulso venoso jugular positivo e à palpação abdominal os animais não apresentaram alterações ou mantiveram o abdômen tenso. No grupo Controle, 33,33\% dos cães manifestaram reflexo de tosse positivo, enquanto nos grupos Estágio B2 e Estágio C estas porcentagens foram de $28,57 \%$ e $80 \%$, respectivamente. Os cães do grupo Controle não possuíam sopro cardíaco, enquanto nos grupos Estágio B2 e Estágio C os animais apresentaram sopro sistólico cujo grau está especificado na Tabela 3. Os grupos não apresentaram diferença estatisticamente significativa entre os valores de frequência respiratória e de temperatura do ambiente onde foi realizada a avaliação (Tabela 4). 
Tabela 3. Descrição do grau dos sopros apresentados pelos animais nos diferentes grupos experimentais.

Grau do sopro cardíaco n $(\%)$

Controle $(\mathrm{n}=6)$

Estágio B2 (n=7)

Estágio C (n=10)

Foco Mitral

\begin{tabular}{llcc}
\hline II/VI & 0 & $1(14,28)$ & 0 \\
\hline III/VI & 0 & $6(85,71)$ & $1(10)$ \\
\hline IV/VI & 0 & 0 & $6(60)$ \\
\hline V/VI & 0 & 0 & $3(30)$ \\
\hline
\end{tabular}

Foco Tricúspide

\begin{tabular}{lccc}
\hline II/VI & 0 & $2(28,57)$ & $1(10)$ \\
\hline III/VI & 0 & $3(42,85)$ & $3(30)$ \\
\hline IV/VI & 0 & 0 & $4(40)$ \\
\hline V/VI & 0 & 0 & $2(20)$ \\
\hline
\end{tabular}

Em relação aos parâmetros clínicos centrais, todos os animais apresentaram pulso femoral forte e sincrônico à palpação. Um animal do grupo Estágio C (10\%) apresentou distensão jugular e outro possuía reflexo hepatojugular positivo (10\%). Não houve diferença estatisticamente significativa entre os valores de frequência cardíaca, temperatura retal e pressão arterial sistêmica sistólica (PAS) entre os grupos (Tabela 4).

À avaliação dos parâmetros clínicos periféricos, todos os animais estavam alertas, apresentaram mucosas róseas e tempo de preenchimento capilar menor que dois segundos. Também não houve diferença estatisticamente significativa entre os grupos para os valores de gradiente entre as temperaturas $(\Delta \mathrm{T})$ central e periférica e periférica e ambiente. A única diferença estatisticamente significativa entre os grupos estudados foi apontada na dosagem de lactato sérico (Tabela 4), onde o grupo Estágio C apresentou níveis de lactato mais elevados comparados aos grupos Controle $(P=0,024)$ e Estágio B2 $(P=0,045)$ (Figura 2). 
Figura 2. Gráfico box-plot representando os níveis de lactato (mmol/L) nos diferentes grupos experimentais.

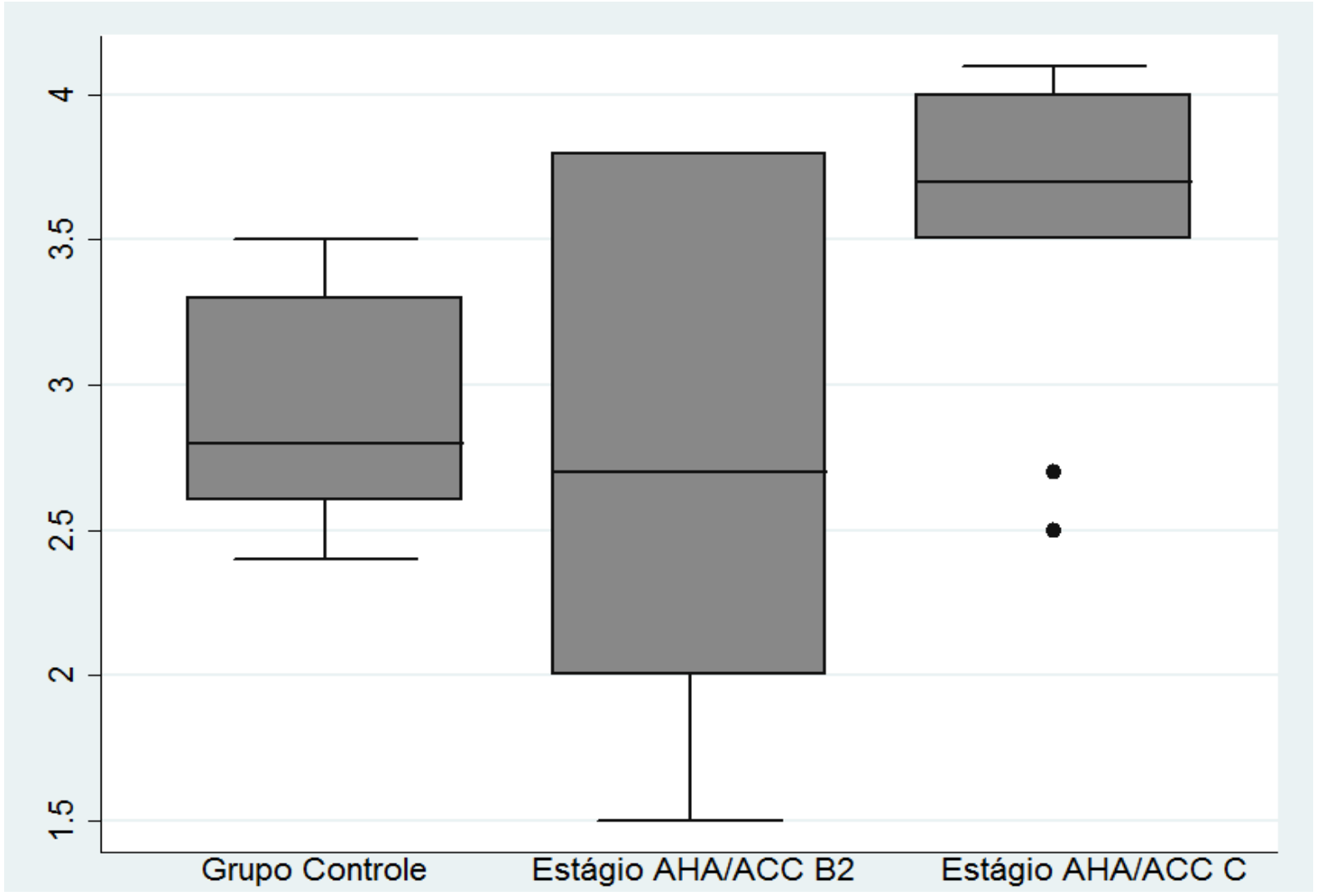

Legenda: AHA/ACC: American Heart Association/American College of Cardiology.

Os grupos não apresentaram diferenças estatisticamente significativas entre os valores de volume globular, contagem de hemácias, hemoglobina, leucócitos e de proteína plasmática total. O único parâmetro hematológico que apresentou diferença estatisticamente significativa entre os grupos foi a contagem de plaquetas, na qual o grupo Controle apresentou diferença significativa em relação ao grupo Estágio $C(P=0,011)$ (Tabela 4). 
Tabela 4. Parâmetros de exame físico, perfusionais e hematológicos nos diferentes grupos experimentais.

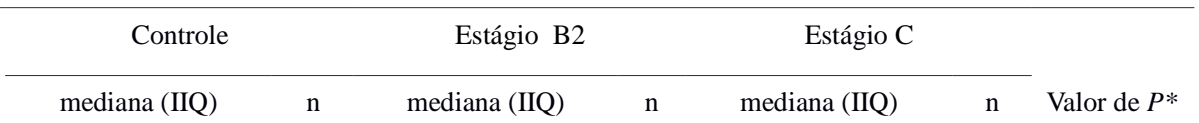

\begin{tabular}{|c|c|c|c|c|c|c|c|}
\hline \multicolumn{8}{|l|}{ Parâmetros Clínicos } \\
\hline Frequência cardíaca (bpm) & $119(98-153) \mathrm{a}$ & 6 & $120(108-152) \mathrm{a}$ & 7 & $142(135-151)$ a & 10 & 0,1436 \\
\hline Temperatura retal $\left({ }^{\circ} \mathrm{C}\right)$ & $38,7(38,28-39,33) \mathrm{a}$ & 6 & $38,9(38,80-39,10) \mathrm{a}$ & 7 & $38,6(38,30-39,03) \mathrm{a}$ & 10 & 0,4518 \\
\hline PAS (mmHg) & $146,6(143-169)$ a & 6 & $145,8(132-154,4)$ a & 7 & $143,8(127,2-151,2) \mathrm{a}$ & 10 & 0,4982 \\
\hline$\Delta \mathrm{T}$ periférica e ambiente $\left({ }^{\circ} \mathrm{C}\right)$ & $9,02(2,63-10,00)$ a & 6 & $6,60(6,23-7,53)$ a & 7 & $7,96(6,16-9,13) \mathrm{a}$ & 10 & 0,3629 \\
\hline Lactato (mmol/L) & $2,80(2,55-3,35) \mathrm{a}$ & 6 & $2,70(2,00-3,80) \mathrm{a}$ & 7 & $3,70(3,30-4,02) b$ & 10 & 0,0385 \\
\hline \multicolumn{8}{|l|}{ Parâmetros hematológicos } \\
\hline Volume globular (\%) & $51(44-56,5)$ a & 6 & $41(36-45) \mathrm{a}$ & 7 & $45,5(34-49,5) \mathrm{a}$ & 10 & 0,1106 \\
\hline 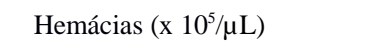 & $7,4(6,45-8,27) \mathrm{a}$ & 6 & $6,46(5,2-6,56) \mathrm{a}$ & 7 & $6,42(5,15-7,27) \mathrm{a}$ & 10 & 0,1064 \\
\hline Hemoglobina (g/dL) & $16,30(14,35-18,75) \mathrm{a}$ & 6 & $14,60(12,40-15,00) \mathrm{a}$ & 7 & $15,15(11,18-16,10)$ a & 10 & 0,2195 \\
\hline
\end{tabular}

Legenda: AHA/ACC: American Heart Association/American College of Cardiology; IIQ: intervalo interquartil; $\Delta \mathrm{T}$ : gradiente de temperatura; PAS: pressão arterial sistêmica sistólica; PPT: proteína plasmática total; a, b: letras iguais indicam ausência de diferença estatisticamente significativa; *: Teste Kruskal-Wallis.

\section{Discussão}

A mensuração do lactato sérico é amplamente utilizada na medicina intensiva como um indicador de perfusão tecidual, já que representa um dos produtos intermediários que está elevado em consequência da reorganização do metabolismo celular durante a hipóxia tecidual (Valenza et al., 2005). Além disso, há evidências que suportam seu uso como marcador de hipoperfusão oculta (Meregalli et al., 2004; Young et al., 2014). Nas situações em que haja 
diminuição do débito cardíaco, a hiperlactatemia provavelmente resulta de metabolismo anaeróbico e de prejuízo no "clearence", provavelmente devido à hipóxia tecidual generalizada (Jakob et al., 2001; Jansen et al., 2009). O lactato é facilmente mensurado quando comparado a outras variáveis de avaliação da perfusão, como a saturação venosa mista de oxigênio $\left(\mathrm{SvO}_{2}\right)$ ou a saturação venosa central de oxigênio $\left(\mathrm{SvcO}_{2}\right)$ que requerem o uso de um catéter venoso profundo no momento do primeiro atendimento, ou mesmo quando confrontados com a punção arterial para medir o déficit de bases, $\mathrm{pH}$ ou o gap de $\mathrm{CO}_{2}$ (Meregalli et al., 2004; Conti-Patara et al., 2012; Hu et al., 2012).

No entanto, a mensuração do lactato tem suas limitações, pois diversas condições podem ocasionar a sua elevação sérica, como as que resultem em hiperlactatemia tipo B; neste estudo o foco foi a hiperlactatemia tipo A provocada por má perfusão (Meregalli et al., 2004; Karagiannis et al., 2006).

Todas estas situações consideradas passíveis de geração da hiperlactatemia tipo B foram examinadas no presente estudo, a fim de se excluir ao máximo possíveis comorbidades que poderiam se comportar como fatores de confusão no momento da sua constatação. Dessa forma, os animais incluídos e que apresentaram hiperlactatemia com o avanço da IC no grupo Estágio $\mathrm{C}$ foram provavelmente associados ao tipo $\mathrm{A}$, atribuída à hipoperfusão e decorrente de hipóxia tecidual global.

Há relatos de que a concentração plasmática de lactato de 3 a $5 \mathrm{mmol} / \mathrm{L}$ corresponderia a hipoperfusão sistêmica leve em cães (Boag e Hughes, 2005; Karagiannis et al., 2006). O valor de referência estabelecido para a mensuração de lactato em cães hígidos utilizando-se o aparelho Accutrend Plus Roche é de 1,2 a 3,1 mmol/L e apenas a mediana do grupo Estágio C estava acima deste limite superior (Stevenson et al., 2007). Para os animais atendidos como urgência independente da doença de origem os níveis de lactato associados a maior sobrevida deveriam estar abaixo de 3,2 mmol/L (Rabelo et al., 2009). 
Neste estudo, o grupo Estágio C apresentou níveis de lactato sérico mais elevados que os grupos Controle e Estágio B2, com significância clínica e estatística entre as diferenças. Estes dados corroboram a hipótese inicial do estudo de que cães com IC possam apresentar hipoperfusão tecidual oculta detectável por meio da avaliação dos níveis séricos de lactato.

Goutal et al. (2010) avaliaram cães e gatos atendidos em um serviço de emergência com ICC em um estudo retrospectivo. Cento e doze animais em classe II do International Small Animal Cardiac Health Council (ISACHC), correspondente ao estágio C da AHA/ACC, apresentaram registro de mensuração de lactato à admissão, 72 (64\%) possuíam níveis de lactato sérico elevados (mediana $=2,5 \mathrm{mmol} / \mathrm{L}$ ) de acordo com o intervalo de referência da instituição (de 0,1 a $1,9 \mathrm{mmol} / \mathrm{L}$ ). No entanto, não foram excluídas as comorbidades que poderiam influenciar na hiperlactatemia, como a doença renal crônica e a IC decorrente de outras doenças, diferentemente dos critérios de exclusão utilizados neste estudo. Ambos os experimentos demonstraram a prevalência de hiperlactatemia em estágios mais avançados de IC.

Em humanos com IC atendidos em serviço de emergência, ficou demonstrado que há diferença estatística e clinicamente significativa na mensuração pontual do lactato sérico após a admissão hospitalar. Mas estes pacientes apresentaram valores de frequência cardíaca e pressão arterial sistêmica inalterados, o que levou os autores a caracterizarem o quadro como déficit de perfusão oculto em pacientes com IC. Neste caso a hiperlactatemia identificou pacientes com quadros mais graves de IC, corroborando os níveis mais elevados do lactato sérico observado no grupo Estágio C, que abrangeu os cães com o quadro mais avançado de IC dentre os incluídos no estudo (Ander et al., 1998; Milzman et al., 1998).

É importante destacar que a hipoperfusão local significativa de certos órgãos, como trato gastrointestinal e pele, pode ocorrer antes da hipoperfusão sistêmica se manifestar clinicamente, por exemplo, na forma de hiperlactatemia e hipotensão (Kreisberg, 1972; 
Bellomo et al., 1996; Kaplan et al., 2001; Boag e Hughes, 2005). Na insuficiência circulatória, o fluxo sanguíneo é dividido entre os tecidos da linha periférica (rins e trato gastrointestinal principalmente, além da pele e outras vísceras) e os órgãos centrais (coração, cérebro e pulmões) a fim de manter a perfusão de órgãos considerados de primeira necessidade em detrimento da circulação periférica. Assim, o monitoramento da perfusão nos órgãos periféricos pode ser um marcador precoce de hipoperfusão (Joly e Weil, 1969; Lima e Bakker, 2005; van Genderen et al., 2012). Em casos de choque circulatório, o aumento da vasoconstrição produz diminuição da temperatura corpórea e habilidade diminuída do core em regular sua temperatura antes que a hipoperfusão ocorra, o que não foi observado em nenhum dos grupos de IC.

Consequentemente, a linha central será mantida às custas da periferia para preservar a perfusão dos órgãos vitais, resultando em aumento do gradiente de temperatura centroperiférico, um indicador de perfusão periférica, em relação ao valor de referência de 3 a $7^{\circ} \mathrm{C}$ (Curley e Smyrnios, 2003; van Genderen et al., 2012). Esta situação foi observada no grupo Estagio B2. A circulação cutânea, por não possuir uma autorregulação própria nos casos de hipoperfusão sistêmica, presente em órgãos vitais, não consegue manter fluxo sanguíneo em estados de baixa pressão. Dessa forma, a resposta neurohumoral simpática predomina na pele, ocasionando o decréscimo na temperatura cutânea em situações de falência circulatória (Lima e Bakker, 2005). Ademais, é essencial que haja controle da temperatura ambiente em todos os estudos que avaliem gradientes de temperatura, conforme foi realizado no presente trabalho, a qual foi mantida na faixa termoneutra em torno de $24^{\circ} \mathrm{C}$, a fim de não influenciar a temperatura periférica (Schey et al., 2009; Schey et al., 2010).

Neste estudo, os animais do grupo Estágio C não apresentaram elevação no valor do gradiente de temperatura centro-periférico como esperado, uma vez que houve elevação no valor de lactato sérico, indicativo de hipoperfusão. Supomos que a administração de 
pimobendan nos pacientes desse grupo tenha inibido a vasoconstrição cutânea, e consequente aumento do gradiente de temperatura centro-periférico, por meio de seu mecanismo de ação. O pimobendan promove vasodilatação por meio da inibição da fosfodiesterase-III nas células da musculatura lisa vascular, ação esta mantida inclusive em cães com ICC grave (Ohte et al., 1997). Além disso é sabido que pacientes com idade avançada podem apresentar uma dificuldade fisiológica de responder com eficiência simpática nos casos de desvio agudo de fluxo entre os diversos leitos, provavelmente por desgaste simpático da idade. E neste estudo os doentes do grupo C eram mais idosos (média \pm desvio-padrão, idade 13,50 \pm 1,84 anos) que os do grupo B2 (média \pm desvio-padrão, idade 11,29 \pm 1,79 anos), com uma importante diferença clínica e estatística $(P=0,039)$.

Nosso trabalho não demonstrou diferença entre os grupos com relação aos parâmetros clínicos como a frequência cardíaca, a pressão arterial sistólica, a qualidade de pulso, o tempo de preenchimento capilar, a coloração de mucosas ou nível de consciência, o que aumenta a possibilidade de existência de hipoperfusão oculta nos cães do grupo Estágio C, já que aqueles animais apresentaram hiperlactatemia importante (Milzman et al., 1998; Ander et al., 1999a; Ander et al., 1999b; Kaplan et al., 2001; Meregalli et al., 2004; Hu et al., 2012).

Em relação aos parâmetros hematológicos, os grupos não demonstraram déficits em relação à contagem de hemácias, hemoglobina ou do volume globular, descartando a anemia como causa da hiperlactatemia (Strehlow, 2010). Houve diferença estatisticamente significativa entre a contagem de plaquetas dos grupos Controle e Estágio C. Embora os valores se encontrassem dentro do intervalo de normalidade para a espécie, observou-se uma tendência de aumento na contagem de plaquetas do grupo Estágio $\mathrm{C}$ (Meinkoth e Clinkenbeard, 2000). Sabe-se que a IC pode ocasionar uma elevação sustentada na concentração sérica de citocinas pró-inflamatórias como IL-1, IL-6, IL-18 e TNF - $\alpha$, o que pode levar a um aumento na concentração de plaquetas (Baker, 2007; Sandhu et al., 2010). O 
aumento destas citocinas também pode justificar a tendência de elevação observada na contagem de leucócitos entre os grupos, bem como a tendência de diminuição na contagem de hemácias, com o grupo C apresentando a maior mediana de leucócitos e a menor de hemácias (Weiser, 2007; Sandhu et al., 2010; Divakaran et al., 2011). A anemia em pacientes com insuficiência cardíaca é multifatorial e uma de suas possíveis causas é a anemia da inflamação, decorrente da supressão da medula óssea e alterações na dinâmica de absorção intestinal de ferro e de seu reaproveitamento por macrófagos, processos estes mediados por citocinas (Sandhu et al., 2010; Divakaran et al., 2011).

Também observou-se uma maior prevalência de machos, possivelmente pela elevada predisposição que este sexo tem em desenvolver a DCVM, bem como pela exclusão de um grande número de fêmeas pela alta prevalência de neoplasia mamária em cadelas idosas, um critério de exclusão deste estudo (Atkins et al., 2009).

Como limitações deste trabalho podemos citar a falta de padronização do tratamento dos animais de um mesmo grupo e entre os grupos, na medida que as abordagens terapêuticas tinham que atender à necessidade clínica de cada paciente. Embora haja uma possível influência do tratamento instituído nos resultados, tal medida mostrou-se eticamente necessária. Não foram utilizados métodos de avaliação direta da perfusão, como a $\mathrm{SvO}_{2}$, $\mathrm{SvcO}_{2}$, déficit de bases, $\mathrm{pH}$ ou o gap de $\mathrm{CO}_{2}$, o que poderia auxiliar na confirmação de hiperlactatemia decorrente de hipoperfusão (tipo A) (Hu et al., 2012). Este estudo foi unicêntrico e com um número reduzido de animais, o que não permitiu que os resultados fossem extrapolados para todos os cães com IC decorrente da DCVM. No entanto, estes dados descritivos podem ser úteis durante a abordargem clínica de pacientes com IC decorrente da DCVM. 


\section{Conclusão}

Conclui-se que cães em IC decorrente de DCVM podem apresentar hipoperfusão tecidual oculta no Estágio C, constatada pela hiperlactatemia. A IC deve ser considerada no diagnóstico diferencial de hiperlactatemia em pacientes com DCVM.

\section{Conflito de interesses}

Nenhum dos autores possui relações pessoais ou financeiras que possam influenciar inapropriadamente ou enviesar o estudo.

\section{Agradecimentos}

Ao Hospital Veterinário de Pequenos Animais da Universidade de Brasília pelo suporte financeiro à pesquisa.

\section{Referências}

Adeva-Andany, M., López-Ojén, M., Funcasta-Calderón, R., Ameneiros-Rodríguez, E., Donapetry-García, C., Vila-Altesor, M., Rodríguez-Seijas, J., 2014. Mitochondrion 17, 76-100.

Ander, D.S., Jaggi, M., Rivers, E., Rady, M.Y., Levine, B., Levine, A.B., Masura, J., Gryzbowski, M., 1998. Undetected cardiogenic shock in patients with congestive heart failure presenting to the emergency department. The American Journal of Cardiology 82, 888-891.

Ander, D.S., Jaggi, M., Rivers, E., 1999a. Identifying occult cardiogenic shock in patients with CHF. Cardiology Review 16, 12-15.

Ander, D.S., Jaggi, M., Rivers, E., 1999b. Case report: patient with chronic severe heart failure and occult shock. Cardiology Review 16, 16.

Atkins, C., Bonagura, J., Ettinger, S., Fox, P., Gordon, S., Haggstrom, J., Hamlin, R., Keene, B., Luis-Fuentes, V., Stepien, R., 2009. Guidelines for the diagnosis and treatment of canine chronic valvular heart disease. Journal of Veterinary Internal Medicine 23, 1142-1150.

Baker, D.C., 2007. Diagnóstico dos distúrbios hemostáticos. In: Hematologia e Bioquímica 
Clínica Veterinária, Primeira Ed. Roca, São Paulo, SP, Brasil, pp. 170-187.

Bellomo, R., Kellum, J.A., Pinsky, M.R., 1996. Transvisceral lactate fluxes during early endotoxemia. Chest 110, 198-204.

Boag, A.K., Hughes, D., 2005. Assessment and treatment of perfusion abnormalities in the emergency patient. Veterinary Clinics of North America: Small Animal Practice 35, 319-342.

Borgarelli, M., Haggstrom, J., 2010. Canine degenerative myxomatous mitral valve disease: natural history, clinical presentation and therapy. Veterinary Clinics of North America: Small Animal Practice 40, 651-663.

Borgarelli, M., Buchanan, J.W., 2012. Historical review, epidemiology and natural history of degenerative mitral valve disease. Jounal of Veterinary Cardiology 14, 93-101.

Boysen, S.R., Bozzetti, M., Rose, L., Dunn, M., Pang, D.S.J., 2009. Effects of prednisone on blood lactate concentrations in healthy dogs. Journal of Veterinary Internal Medicine $23,1123-1125$.

Brown, S., Atkins, C, Bagley, R., Carr, A., Cowgill, L., Davidson, M., Egner, B., Elliott, J., Henik, R., Labato, M. et al., 2007. Guidelines for the identification, evaluation, and management of systemic hypertension in dogs and cats. Journal of Veterinary Internal Medicine 21, 542-558.

Conti-Patara, A., Caldeira, J.A., Mattos-Junior, E., Carvalho, H.S., Reinoldes, A., Pedron, B.G., Patara, M., Talib, M.S.F., Faustino, M., Oliveira, C.M., Cortopassi, S.R.G., 2012. Changes in tissue perfusion parameters in dogs with severe sepsis/septic shock in response to goal-directed hemodynamic optimization at admission to ICU and the relation to outcome. Journal of Veterinary Emergency and Critical Care 00, 1-10.

Curley, F.J., Smyrnios, N.A., 2003. Routine monitoring of critically ill patients. In: Intensive Care Medicine, Fifth Ed. Lippincott Williams \& Wilkins, Baltimore, MD, USA, pp. 250-270.

Divakaran, V., Mehta, S., Yao, D., Hassan, S., Simpson, S., Wiegerinck, E., Swinkels, D.W., Mann, D.L., Afshar-Kharghan, V., 2011. Hepcidin in anemia of chronic heart failure. American Journal of Hematology 86, 107-109.

Goldston, R.T., Hoskins, J.D., 1999. In: Geriatria e gerontologia em cães e gatos, Roca, São Paulo, SP, Brasil. 551 p.

Goutal, C.M., Keir, I., Kenney, S., Rush, J.E., Freeman, L.M., 2010. Evaluation of acute congestive heart failure in dogs and cats: 145 cats (2007-2008). Journal of Veterinary Emergency and Critical Care 20, 330-337.

Hu, B.Y., Laine, G.A., Wang, S., Solis, R.T., 2012. Combined central venous oxygen saturation and lactate as markers os occult hypoperfusion and outcome following cardiaca surgery. Journal of Cardiothoracic and Vascular Anesthesia 26, 52-57. 
Jakob, S.M., Tenhunen, J.J., Laitinen, S., Heino, A., Alhava, A., Takala, J., 2001. Effects of systemic arterial hypoperfusion on splanchnic hemodynamics and hepatic arterial buffer response in pigs. American Journal of Physiology-Gastrointestinal and Liver Physiology 280, G819-827.

Jansen, T.C., van Bommel, J., Bakker, J., 2009. Blood lactate monitoring in critically ill patients: a systematic health technology assessment. Critical Care Medicine 37, 28272839.

Joly, H.R.; Weil, M.R., 1969. Temperature of the great toe as an indication of the severity of shock. Circulation 39, 131-138.

Kaplan, L.J., McPartland, K., Santora, T.A., Trooskin, S.Z., 2001. Start with a subjective assessment of skin temperature to identify hypoperfusion in intensive care unit patients. The Journal of Trauma and Acute Care Surgery 50, 620-628.

Karagiannis, M.H., Reniker, A.N., Kerl, M.E., Mann F.A., 2006. Lactate measurement as an indicator of perfusion. Compendium on Continuing Education for the Practicing Veterinarian 28, 287-298.

Kienle, R.D., 1998. Echocardiography. In: Small Animal Cardiovascular Medicine, First Ed. Mosby, St. Louis, MO, USA, pp. 95-117.

Kittleson, M.D., Brown, W.A., 2003. Regurgitant fraction measured by using the proximal isovelocity surface area method in dogs with chronic myxomatous mitral valve disease. Journal of Veterinary Internal Medicine 17, 84-88.

Kreisberg, R.A., 1972. Glucose-lactate inter-relations in man. New England Journal of Medicine 287, 132-137.

Lima, A., Bakker, J., 2005. Noninvasive monitoring of peripheral perfusion. Intensive Care Medicine 31, 1316-1326.

Lima, A.F.K.T., Repetti, C.S.F., Hataka, A., Franco, R.P., 2010. Valores do lactato sérico em um cão com mastocitoma grau III - Relato de caso. Medvep 8, 600-604.

Meinkoth, J.H., Clinkenbeard, K.D., 2000. Normal Hematology of the dog. In: Schalm's Veterinary Hematology, Fifth Ed. Lippincott Williams \& Wilkins, Baltimore, MD, USA, pp. 1057-1063.

Meregalli, A., Oliveira, R.P., Friedman, G., 2004. Occult hypoperfusion is associated with increased mortality in hemodynamically stable, high-risk, surgical patients. Critical Care 8, R60-R65.

Milzman, D., Moskowitz, L., Sadammar, R., Strudwick, W., Williams, J., Clement, M., 1998. Clinical utility of rapid lactate determination to identify occult perfusion deficits in heart failure patients. Journal of Cardiac Failure 4, 36. 
Ohte, N., Cheng, C.P., Suzuki, M., Little, W.C., 1997. The cardiac effects of pimobendan but not amrinone) are preserved at rest and during exercise in conscious dogs with pacinginduced heart failure. The Journal of Pharmacology and Experimental Therapeutics 282, 23-31.

Olsen, L.H., Häggström, J., Petersen, H.D., 2010. Acquired valvular heart disease. In: Textbook of Veterinary Internal Medicine, Seventh Ed. Saunders Elsevier, St. Louis, MO, USA, pp. 1299-1319.

Rabelo R.C., Fragio C., Alsua S., 2009. RICO Score: Classificação rápida de sobrevida em cuidados intensivos. Variáveis inter-relacionadas em cães. Clínica Veterinária 78, 28 38 .

Rabelo, R.C., 2010. Métodos de avaliação da perfusão no paciente grave. Jounal Latinoamericano de Medicina Veterinaria de Emergencia y Cuidados Intensivos 2, 134-154.

Sandhu, A., Soman, S., Hudson, M., Besarab, A., 2010. Managing anemia in patients with chronic heart failure: what do we know?. Vascular Health and Risk Management 6, 237-252.

Schey, B.M., Williams, D.Y., Bucknall, T., 2009. Skin temperature as a noninvasive marker of haemodynamic and perfusion status in adult cardiac surgical patients: an observational study. Intensive and Critical Care Nursing 25, 31-37.

Schey, B.M., Williams, D.Y., Bucknall, T., 2010. Skin temperature and core-peripheral temperature gradient as markers of hemodynamic status in critically ill patients: a review. Heart \& Lung 39, 27-40.

Sisson, D.D., 2010. Pathophysiology of heart failure. In: Textbook of Veterinary Internal Medicine, Seventh Ed. Saunders Elsevier, St. Louis, MO, USA, pp. 1143-1158.

Stevenson, C.K., Kidney, B.A., Duke, T., Snead, E.C.R., Jackson, M.L., 2007. Evaluation of the Accutrend for lactate measurement in dogs. Veterinary Clinical Pathology 36, 261266.

Strehlow, M.C., 2010. Early identification of shock in critically ill patients. Emergency Medicine Clinics of North America 28, 57-66.

Valenza, F., Aletti, G., Fossali, T., Chevallard, G., Sacconi, F., Irace, M., Gattinoni, L., 2005. Lactate as a marker of energy failure in critically ill patients: hypothesi. Critical Care 9, 588-593.

van Genderen, M.E., van Bommel, J., Lima, A., 2012. Monitoring peripheral perfusion in critically ill patients at the bedside. Current Opinion in Critical Care 18, 273-279.

van Hall, G., 2010. Lactate kinetics in human tissues at rest and during exercise. Acta Physiologica 199, 499-508. 
Waddell, L.S., 2010. Hypotension. In: Textbook of Veterinary Internal Medicine, Seventh Ed. Saunders Elsevier, St. Louis, MO, USA, pp. 585-588.

Weiser, G., 2007. Interpretação da resposta leucocitária nas doenças. In: Hematologia e Bioquímica Clínica Veterinária, Primeira Ed. Roca, São Paulo, SP, Brasil, pp. 127140.

Young, B.C., Prittie, J.E., Fox, P., Barton, L.J., 2014. Decreased central venous oxygen saturation despite normalization of heart rate and blood pressure post shock resuscitation in sick dogs. Journal of Veterinary Emergency and Critical Care 24, 154161. 


\section{ANEXO A - ESTADIAMENTO CLÍNICO DA IC BASEADO NO SISTEMA MODIFICADO DA AHA/ACC (Atkins et al., 2009)}

\begin{tabular}{|c|l|}
\hline Estágio & \multicolumn{1}{|c|}{ Descrição } \\
\hline A & $\begin{array}{l}\text { Pacientes sob alto risco de desenvolvimento de doença } \\
\text { cardíaca, mas que não possuem desordem estrutural cardíaca } \\
\text { identificável (eg. raça predisposta com ausência de sopro } \\
\text { cardíaco) }\end{array}$ \\
\hline Bacientes assintomáticos em relação a manifestações clínicas \\
de IC, com doença cardíaca estrutural (presença de sopro \\
cardíaco), porém sem evidências radiográficas e/ou \\
ecocardiográficas de remodelamento cardíaco decorrente da \\
degeneração valvar (eg. aumento do diâmetro de câmaras \\
cardíacas esquerdas)
\end{tabular}


ANEXO B - DECLARAÇÃO DA COMISSÃO DE ÉTICA NO USO ANIMAL

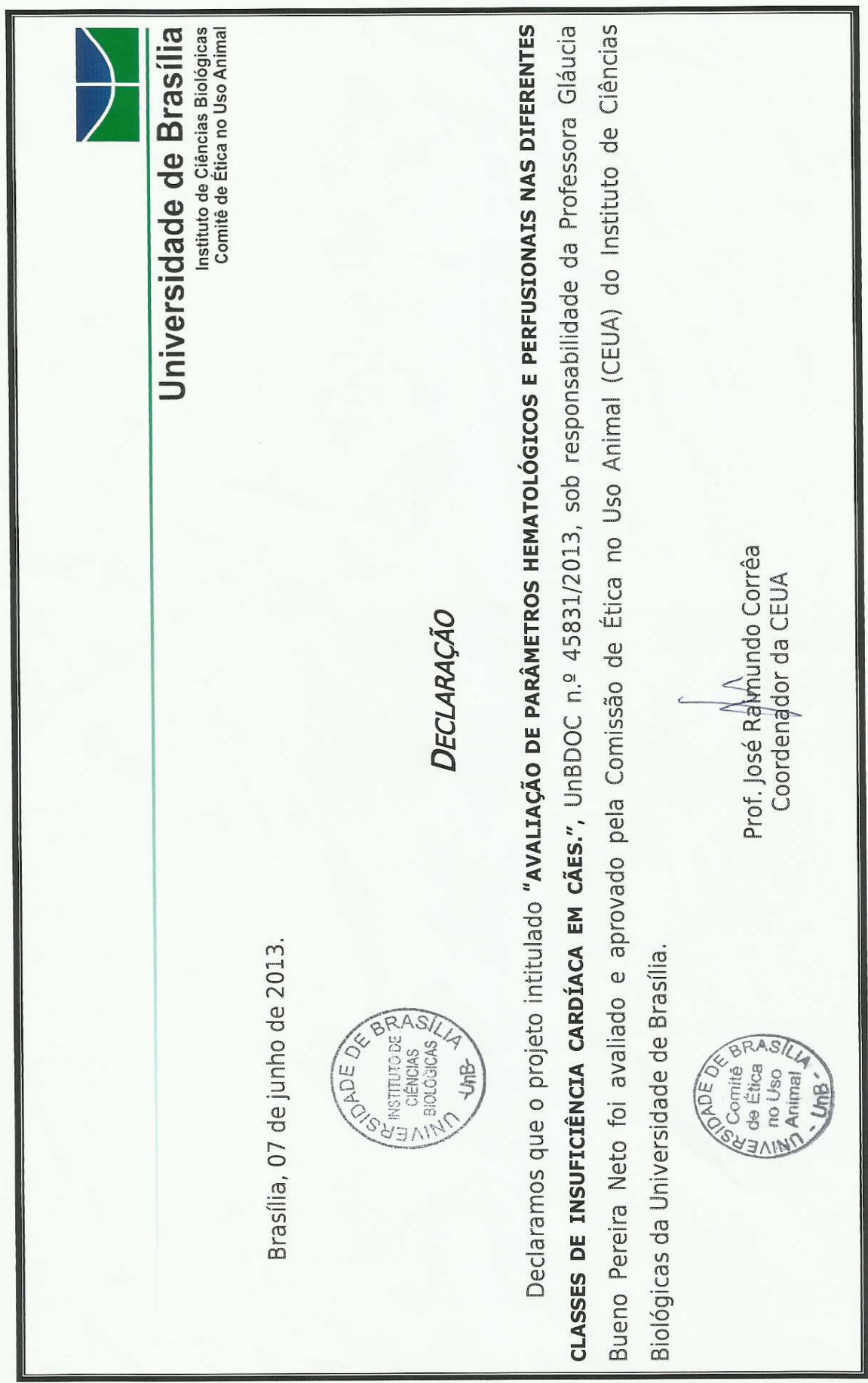

